\title{
Une confirmation : le produit du gène ob est bien une hormone agissant comme un lipostat
}

Il y a quelques mois, nous rapportions le clonage du gène $o b$, responsable, à l'état homozygote, d'une obésité génétique de la souris $(\mathrm{m} / \mathrm{s}$ $n^{\circ} 12$, vol. $10, p$. 1337). Ce gène, exprimé dans le tissu adipeux, code pour une protéine de $16 \mathrm{kDa}$ qui a toutes les caractéristiques d'une protéine sécrétée. Le fait que l'obésité de la souris $o b / o b$ puisse régresser en cas de circulation croisée avec une souris normale ou avec une souris souffrant d'une autre forme d'obésité $(d b / d b)$ permettait à Jeffrey Friedman (Rockfeller University, New York, USA) et à ses collègues de suggérer que la protéine $\mathrm{Ob}$ pouvait être une hormone de la satiété, agissant peut-être au niveau hypothalamique, et dont le récepteur pourrait être codé ou contrôlé par le gène $d b(\mathrm{~m} / \mathrm{s}$ $n^{\circ} 12$, vol. 10, p. 1337).

Une série d'expériences rapportées par l'équipe de Friedman [1] ainsi que par des chercheurs de la firme de biotechnologie AMGEN (Thousand Oaks, CA, USA) [2] et de Hoffmann Laroche (Nutley, NJ, USA et Gand, Belgique) [3] confirme la première de ces hypothèses et est en faveur de la seconde. L'injection quotidienne de la protéine $\mathrm{Ob}$ recombinée à des souris mutantes obèses $o b / o b$ tend à normaliser leur poids en quelques semaines. En revanche, ce traitement est inefficace chez des souris obèses $d b / d b$. Le pouvoir amaigrissant de la protéine $\mathrm{Ob}$ se manifeste également sur des souris normales qui perdent pratiquement la totalité de leur tissu adipeux. Des souris souffrant d'une obésité induite par un régime riche en calories sont également sensibles à cette protéine, de même qu'une lignée de souris présentant une obésité de la maturité. L'amaigrisement est dû à un double phénomène : une diminution de l'appétit, comme on peut s'y attendre pour une hormone de la satiété ; et une augmentation de la consommation d'énergie, aboutissant à une mobilisation des graisses. Comme nous l'indiquions dans la précédente nouvelle consacrée à ce sujet, les perspectives commerciales d'un médicament de l'obésité sont tout à fait considérables, expliquant la somme de 20 millions de dollars versée par la société AMGEN pour obtenir une licence exclusive du développement de produits thérapeutiques issus de la connaissance du gène $o b / o b$. L'intérêt d'une hormone du type de la protéine $\mathrm{Ob}$ est, comme le montrent les expériences rapportées par les chercheurs de l'équipe de Friedman et des sociétés AMGEN et Hoffmann Laroche, qu'elle peut être efficace quelle que soit la cause de l'obésité, à l'exception, naturellement, des situations où le récepteur ou la machinerie de transmission du signal de la protéine Ob serait modifié, comme c'est probablement le cas chez des souris $d b / d b$. Les résultats obtenus par les équipes américaines l'ont été avec les protéines murines et humaines. Chez l'homme, les études génétiques ont indiqué que des obésités liées à une modification du gène $o b$ étaient, soit très rares, soit inexistantes. En fait, dans la majorité des cas, les adipocytes des sujets obèses semblent synthétiser une grande quantité de protéine $\mathrm{Ob}$.

Cela est plutôt une bonne nouvelle quant aux perspectives d'utilisation thérapeutique de la protéine $\mathrm{Ob}$ : les obèses synthétisent une protéine $\mathrm{Ob}$ endogène, si bien que l'injection d'une protéine humaine recombinante ne devrait pas entraîner de réaction immune ruinant, à terme, l'efficacité du traitement. Restent naturellement à vérifier l'efficacité et l'innocuité, à moyen et long terme, d'une administration répétée de la protéine $\mathrm{Ob}$ chez l'homme, à affiner la posologie et la pharmacocinétique du produit et à déterminer les meilleures voies d'administration. Seuls ces essais cliniques diront ainsi quelle est l'efficacité de l'injection d'une très grande quantité de protéine $\mathrm{Ob}$ chez des obèses la syn thétisant déjà abondamment, et si des phénomènes de "désensibilisation" des récepteurs ne viendront pas ruiner l'efficacité thérapeutique de l'hormone. Il faut remarquer que, comme chaque fois qu'est envisagé un traitement prolongé par un médicament protéique, la question se posera peutêtre dans le futur du choix entre l'injection répétée de la protéine recombinante et l'implantation de cellules génétiquement modifiées synthétisant et délivrant in situ cette protéine. 
Une troisième voie thérapeutique serait, classiquement, d'administrer per os des inducteurs de l'expression du gène $o b$ endogène. Certains sont d'ailleurs déjà connus, même si leurs autres effets en empêchent évidemment l'utilisation chez les obèses : les glucocorticoïdes. De Vos et al., de l'Institut de Biologie de Lille (France), montrent en effet que, au moins chez le rat, les glucocorticoïdes ont un effet catabolique et amaigrissant corrélé à une augmentation de l'expression du gène $o b[4]$. Nul doute que d'autres molécules inductrices, ou des stéroïdes modifiés dénués d'effets secondaires et conservant leur action sur le gène $o b$ sont, d'ores et déjà, activement recherchés par plusieurs firmes pharmaceutiques, tant il est évident qu'un médicament "classique" pris par voie orale, qu'il s'agisse d'un agoniste du récepteur $\beta_{\mathrm{s}}$ [5] ou d'un inducteur de la synthèse de la leptine (produit du gène $o b$ ) aurait de nombreux avantages sur une protéine ou un ADN thérapeutique

\section{A. Kahn}

Directeur de l'Inserm U. 129. ICGM, unité de génétique et de pathologie moléculaires, Inserm U. 129. 24, rue du FaubourgSaint-Jacques, 75014 Paris, France.

\section{RÉFÉRENCES}

1. Halaas JL, Gajiwala KS, Maffei M, Cohen SL, Chait BT, Rabinowitz D, Lallone RL, Burley SK, Friedman JM. Weight-reducing effects of the plasma protein encoded by the obese gene. Science $1995 ; 269: 543-6$.

2. Pelleymounter MA, Cullen MJ, Baker MB, Hecht R, Winters D, Boone T, Collins F. Effects of the obese gene product on body weight regulation in $o b / o b$ mice. Srience $1995 ; 269: 540-3$.

3. Campfield LA, Smith FJ, Guisez Y, Devos R, Burn P. Recombinant mouse Ob protein: evidence for a peripheral signal linking adiposity and central neural networks. Science $1995 ; 269: 546-9$.

4. De Vos P, Saladin R, Auwerx J, Staels B. Induction of the $a b$ gene expression by corticosteroids is accompanied by body weight loss and reduced food intake. J Biol Chem $1995 ; 270: 15958-61$.

5. Strosberg $\mathrm{AD}$, Manning $\mathrm{BS}^{\prime} \mathrm{J}$. Le récepteur $\beta_{3}$ adrénergique : un gène de poids. médecine/sciences $1995 ; 11$ : 1460-2.

\section{TIRÉS À PART}

